\title{
Properties of the Band Gaps in $1 D$ Ternary Lossy Photonic Crystal Containing Double-Negative Materials
}

\author{
Alireza Aghajamali, ${ }^{1}$ Maryam Akbarimoosavi, $^{2}$ and Mahmood Barati ${ }^{3}$ \\ ${ }^{1}$ Department of Physics, Islamic Azad University, Marvdasht Branch, Marvdasht, Iran \\ ${ }^{2}$ Department of Physics, Shiraz University, Shiraz, Iran \\ ${ }^{3}$ Department of Physics, Islamic Azad University, Science and Research Branch, Fars, Iran
}

Correspondence should be addressed to Alireza Aghajamali; alireza.aghajamali@fsriau.ac.ir

Received 4 September 2013; Revised 27 December 2013; Accepted 29 December 2013; Published 13 February 2014

Academic Editor: José Luís Santos

Copyright (C) 2014 Alireza Aghajamali et al. This is an open access article distributed under the Creative Commons Attribution License, which permits unrestricted use, distribution, and reproduction in any medium, provided the original work is properly cited.

\begin{abstract}
Theoretically, the characteristics matrix method is employed to investigate and compare the properties of the band gaps of the one-dimensional ternary and binary lossy photonic crystals which are composed of double-negative and double-positive materials. This study shows that by varying the angle of incidence, the band gaps for TM and TE waves behave differently in both ternary and binary lossy structures. The results demonstrate that, by increasing the angle of incidence for the TE wave, the width and the depth of zero- $\bar{n}$, zero- $\mu$, and Bragg gap increase in both ternary and binary structures. On the other hand, the enhancement of the angle of incidence for the TM wave contributes to reduction of the width and the depth of the zero- $\bar{n}$ and Bragg gaps, and they finally disappear for incidence angles greater than $50^{\circ}$ and $60^{\circ}$ for the binary structure and $40^{\circ}$ and $45^{\circ}$ for the ternary structures, respectively. In addition, the details of the edges of the band gaps variations as a function of incidence angle for both structures are studied.
\end{abstract}

\section{Introduction}

Photonic crystals (PCs) are artificial dielectric or metallic structures in which the refractive index changes periodically. The periodic structure of the PCs contributes to photonic band gap (forbidden range of frequencies). Interference of the Bragg scattering is considered as a cause of these phenomena, the so-called Bragg gap. PCs have attracted much interest due to their novel electromagnetic waves characteristics and important scientific and engineering applications and have received attentions by many researchers in recent decades [1-8]. With possibility of producing metamaterials, PCs containing metamaterials, namely, "Metamaterial Photonic Crystals" (MPCs), have been made. The technological relevance of the MPCs is of great importance. These structures have attracted considerable attention for their various applications. Designing dielectric mirrors, effective waveguides, filters, and perfect lenses are clear-cut examples. The metamaterials are utilized to design one-dimensional (1D) binary and ternary MPCs with layers of double-negative (DNG) and double-positive (DPS) materials. In these structures, various band gaps can be seen in the transmission spectrum. When the average refractive index of the MPC is equal to zero, the new band gap which is called zero- $\bar{n}$ gap appears in the transmission [9], which possesses some unique properties that distinguish itself from an ordinary Bragg gap. The properties and characteristics of the zero $\bar{n}$ gap of $1 \mathrm{D}$ binary PC structure consisting of DNG and DPS materials were investigated in recent years, such as bandwidth, the depth, and the central frequency of the gap [9-15].

In $1 \mathrm{D}$ binary MPC, in addition to the zero- $\bar{n}$ and Bragg gaps, new gaps appear in transmission spectra for both TE and TM polarized waves. In the TE mode, this gap appears at the frequency where permeability of DNG material vanishes, called the zero- $\mu$ gap, but in the TM mode, this gap arises at the frequency where permittivity of DNG material goes to zero, called the zero- $\varepsilon$ gap. These two new types of gaps are found for the oblique incidence angles but are absent for normal incidence [16-18].

The novel idea of this research is to include the loss factor which is an unavoidable consequence of doublenegative materials to investigate optical properties of $1 \mathrm{D}$ 
binary and ternary MPCs. In this regard, the transmission of electromagnetic waves through a $1 \mathrm{D}$ ternary lossy PC consisting of layers with double-negative and double-positive materials is studied. The effects of the incidence angle and polarization on the characteristics of the zero- $\bar{n}$, zero- $\mu$, zero- $\varepsilon$, and Bragg gaps are investigated. Furthermore, the results are compared with the similar results in the $1 \mathrm{D}$ binary lossy PC. The paper is organized as follows: the MPC structure's design, the permittivity and permeability of the DNG layer, and theoretical formulation (characteristic matrix method) are described in Section 2, the numerical results and discussions are presented in Section 3, and the paper is concluded in Section 4.

\section{Structures Design and Characteristic Matrix Method}

The ID MPC structure under study which is located in air is constituted by alternative layers of DNG and DPS materials, where the DNG material is dispersive and dissipative. We consider the 1D binary MPC with periodic structure of $(A B)^{N}$ and the $1 \mathrm{D}$ ternary MPC with periodic structure of $(A B C)^{N}$, where $A, B$, and $C$ denote three different materials. $N$ is number of the lattice periods, and $d_{i}, \varepsilon_{i}, \mu_{i}$, and $n_{i}(i=A, B, C)$ are thickness, permittivity, permeability, and refractive index of the layers, respectively.

The permittivity and permeability of the DNG (layer $A$ ) in the microwave region are complex and are given as $[9,10,19-$ 21]

$$
\begin{gathered}
\varepsilon_{A}(f)=1+\frac{5^{2}}{0.9^{2}-f^{2}-i f \gamma}+\frac{10^{2}}{11.5^{2}-f^{2}-i f \gamma}, \\
\mu_{A}(f)=1+\frac{3^{2}}{0.902^{2}-f^{2}-i f \gamma},
\end{gathered}
$$

where $f$ and $\gamma$ are frequency and damping frequency, respectively, given in $\mathrm{GHz}$. Various details of the real parts of the permittivity, permeability, and refractive index of layers $A, \varepsilon_{A}^{\prime}, \mu_{A}^{\prime}$, and $n_{A}^{\prime}$ versus frequency for $\gamma=0.2 \times 10^{-3} \mathrm{GHz}$ are shown in Figure 1 . As seen from the figure, there exist three different regions. In the first region, $f<3.13 \mathrm{GHz}, \varepsilon_{A}^{\prime}$ and $\mu_{A}^{\prime}$ simultaneously are negative (DNG material) and $n_{A}^{\prime}<0$. For $3.13<f<3.78 \mathrm{GHz}$, in the second region, $\varepsilon_{A}^{\prime}<0$, but $\mu_{A}^{\prime}>0$ (single-negative material extending to the epsilonnegative material), and similar to the first region, $n_{A}^{\prime}<0$ again. Finally, in the third region, $f>3.78 \mathrm{GHz}$, both $\varepsilon_{A}^{\prime}$ and $\mu_{A}^{\prime}$ are positive (DPS material) and $n_{A}^{\prime}>0$.

The calculation is based on the characteristic matrix method [22], which is most effective to analyze the transmission properties of PCs. The characteristic matrix $M[d]$ for TE wave at incidence angle $\theta_{0}$ from vacuum to a $1 D$ PC structure is given by [22]

$$
M[d]=\prod_{i=1}^{k}\left[\begin{array}{cc}
\cos \gamma_{i} & \frac{-i}{p_{i}} \sin \gamma_{i} \\
-i p_{i} \sin \gamma_{i} & \cos \gamma_{i}
\end{array}\right]
$$

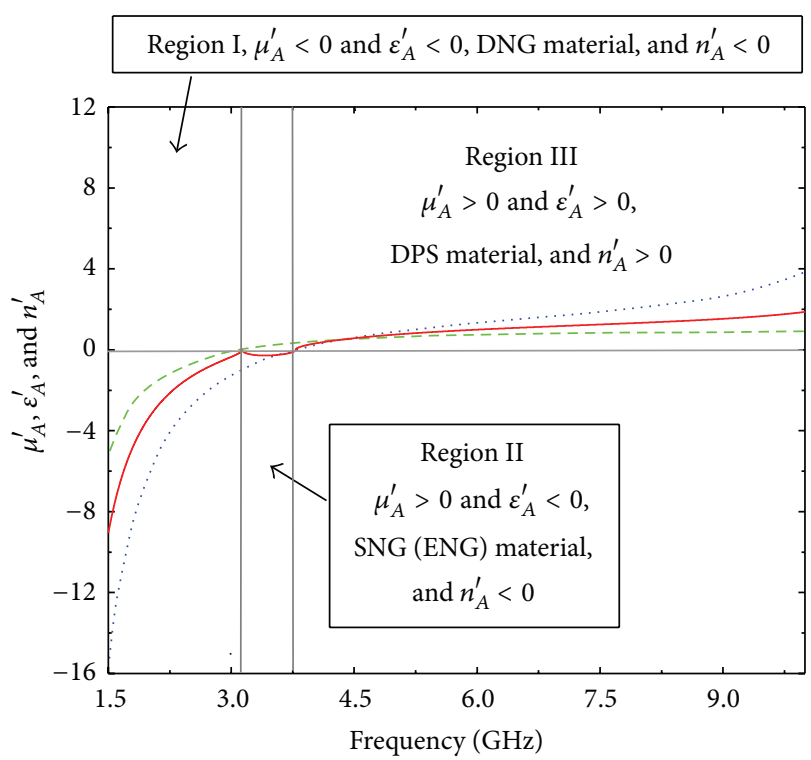

FIGURE 1: Real parts of permittivity (dotted line), permeability (dashed line), and refractive index (solid line) of layers $A, \varepsilon_{A}^{\prime}, \mu_{A}^{\prime}$, and $n_{A}^{\prime}$ versus frequency for a small loss factor.

where $k=2$ and 3 for binary and ternary PCs, respectively. $\gamma_{i}=(\omega / c) n_{i} d_{i} \cos \theta_{i}$, where $c$ is speed of light in vacuum, $\theta_{i}$ is the angle of refraction inside the layer with refractive index $n_{i}$, and $p_{i}$ is given as

$$
p_{i}=\sqrt{\frac{\varepsilon_{i}}{\mu_{i}}} \cos \theta_{i}
$$

where

$$
\cos \theta_{i}=\sqrt{1-\frac{n_{0}^{2} \sin ^{2} \theta_{0}}{n_{i}^{2}}}
$$

The characteristic matrix for $N$ period structure is therefore $[M(d)]^{N}$. The transmission coefficient of the multilayer is given by

$$
t=\frac{2 p_{0}}{\left(m_{11}+m_{12} p_{s}\right) p_{0}+\left(m_{21}+m_{22} p_{s}\right)},
$$

where $m_{i j}(i, j=1,2)$ are the matrix elements of $[M(d)]^{N}$ and

$$
p_{0}=n_{0} \cos \theta_{i}, \quad p_{s}=n_{s} \cos \theta_{s} .
$$

The transmissivity of the multilayer is given by

$$
T=\frac{p_{s}}{p_{0}}|t|^{2}
$$

The above formulations can be applied to TM wave by simple replacements of $p_{i}, p_{0}$, and $p_{s}$ as follows:

$$
\begin{gathered}
p_{i}=\sqrt{\frac{\mu_{i}}{\varepsilon_{i}}} \cos \theta_{i}, \\
p_{0}=\frac{\cos \theta_{0}}{n_{0}}, \quad p_{s}=\frac{\cos \theta_{s}}{n_{s}} .
\end{gathered}
$$




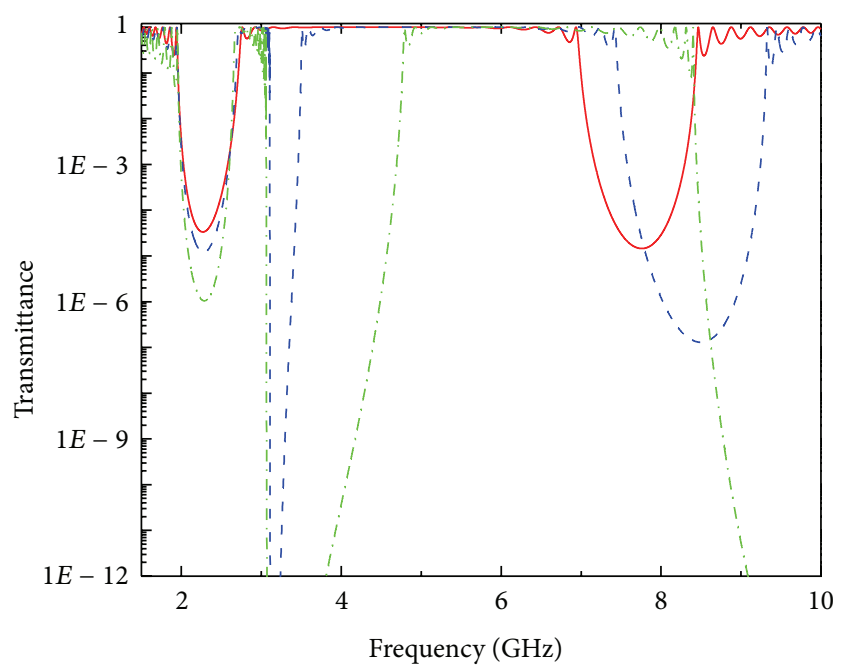

$$
\begin{aligned}
& \text { TE mode } \\
& -\theta_{0}=0^{\circ} \\
& --\theta_{0}=30^{\circ} \\
& --\theta_{0}=60^{\circ}
\end{aligned}
$$

(a)

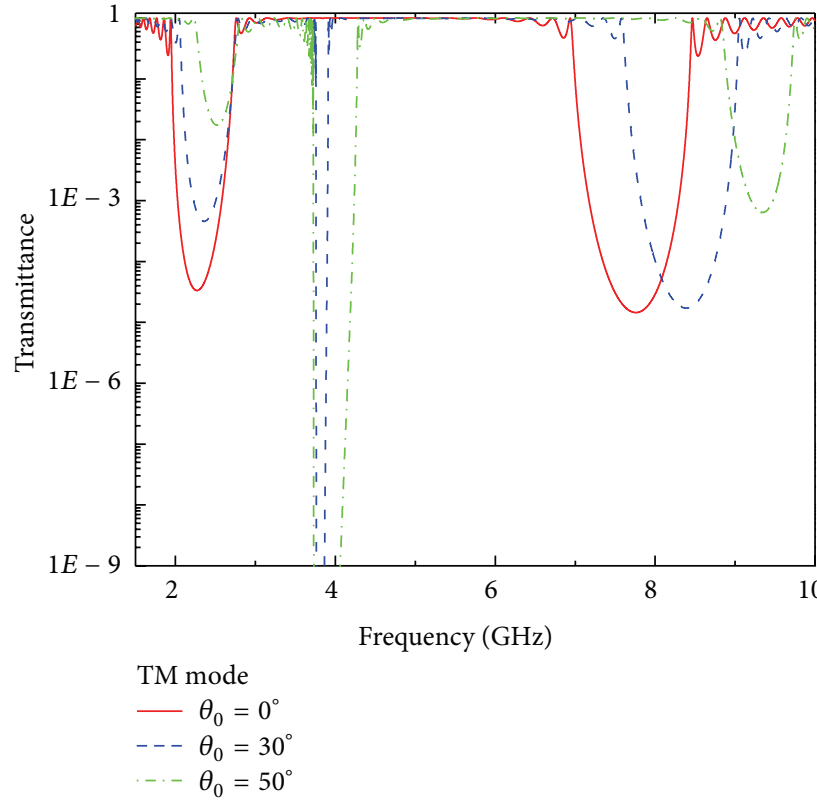

(b)

FIGURE 2: Transmission spectra for the 1D binary MPC structure for different angles of incidence with $\gamma=0.2 \times 10^{-3} \mathrm{GHz}$ : (a) TE and (b) TM waves.

\section{Numerical Results and Discussion}

Based on the theoretical model described in the previous section, the transmission spectrum of the presented lossy MPC structures was calculated. In the study of the $1 D$ binary MPC, consisting of DNG (layer $A$ ) and DPS (layer $B$ ) materials, equations (1) and (2) are used for the permittivity, $\varepsilon_{A}$, and the permeability, $\mu_{A}$, of layer $A$. Layer $B$ is assumed to be a vacuum layer with $\varepsilon_{B}=\mu_{B}=1\left(n_{B}=1\right)$. The thickness of layers $A$ and $B$ is chosen as $d_{A}=6 \mathrm{~mm}$ and $d_{B}=12 \mathrm{~mm}$, respectively, and the total number of lattice periods is set as $N=16[10]$.

The transmission spectra of TE and TM polarized waves for the binary structure at various angles of incidence and for $\gamma=0.2 \times 10^{-3} \mathrm{GHz}$ are shown in Figures 2(a) and 2(b), respectively. For oblique incidence, the zero- $\mu$ and zero- $\varepsilon$ gaps appear in the transmission spectrum for TE and TM waves, respectively, as reported in $[17,18]$. The gaps appear at the frequencies where the sign of the permeability or permittivity of DNG materials changes. As it is seen in Figure 2, the width of the gaps increases as the incidence angle increases, while the left edge of the gaps remains nearly unchanged. In addition, for TE waves, the width and the depth of the zero- $\bar{n}$ and the Bragg gap increase as the incidence angle increases, as reported in [9-14]. On the contrary, for TM waves, the width and the depth decrease as the incidence angle increases (Figure 2(b)). Moreover, the figures show that the central frequencies of the zero- $\bar{n}$, as reported in our previous work [10], and the Bragg gap shift to the higher frequencies as the angle of incidence increases.

In the next part, the band gaps of the $1 \mathrm{D}$ ternary MPC are investigated. The binary structure used before is modified by introducing a third layer of $\mathrm{SiO}_{2}$ (layer $C$ ) with refractive index of $n_{C}=1.46$ and thickness of $d_{C}=6 \mathrm{~mm}$ in each lattice period. The other parameters are kept the same as in the binary structure. The transmission spectra of TE and TM polarized waves for the ternary structure at various angles of incidence and for $\gamma=0.2 \times 10^{-3} \mathrm{GHz}$ are shown in Figures $3(\mathrm{a})$ and 3(b), respectively. As it is seen, the width of the zero$\varepsilon$ and zero- $\mu$ gaps increases by increasing the incidence angle. Although, for TE wave, the width and the depth of the zero- $\bar{n}$ and the Bragg gaps increase as the incidence angles increases. For TM waves, the behaviour is completely different such that the width and the depth of the band gaps decrease when the angle of incidence increases. However, the central frequency of the zero- $\bar{n}$ and Bragg gaps behaves the same as in the binary structure where they shift to the higher frequencies as the angle of incidence increases.

In this part, the band gaps in binary and ternary structures are compared. As it is seen from Figures 2 and 3, the width of the band gaps decreases, when another dielectric layer is added to the lattice period of binary structure. Furthermore, the zero- $\bar{n}$ and Bragg gaps frequencies of the ternary structure are lower than the corresponding frequencies in the binary structures, while the zero- $\varepsilon$ and zero- $\mu$ gaps appear in the same frequencies in both structures.

The lower $\left(f_{L}\right)$ and higher $\left(f_{H}\right)$ frequencies of the zero$\bar{n}$ gap in the binary and ternary structures, as a function of incidence angle for TE and TM waves, are shown in Figures 4(a) and 4(b), respectively. As it is clearly seen, the lower frequency of the gap for the ternary structure is smaller than that in the binary structure for both TE and TM modes for different incidence angles. It is also interesting to note that the width of the gap in the ternary structure for TE wave 


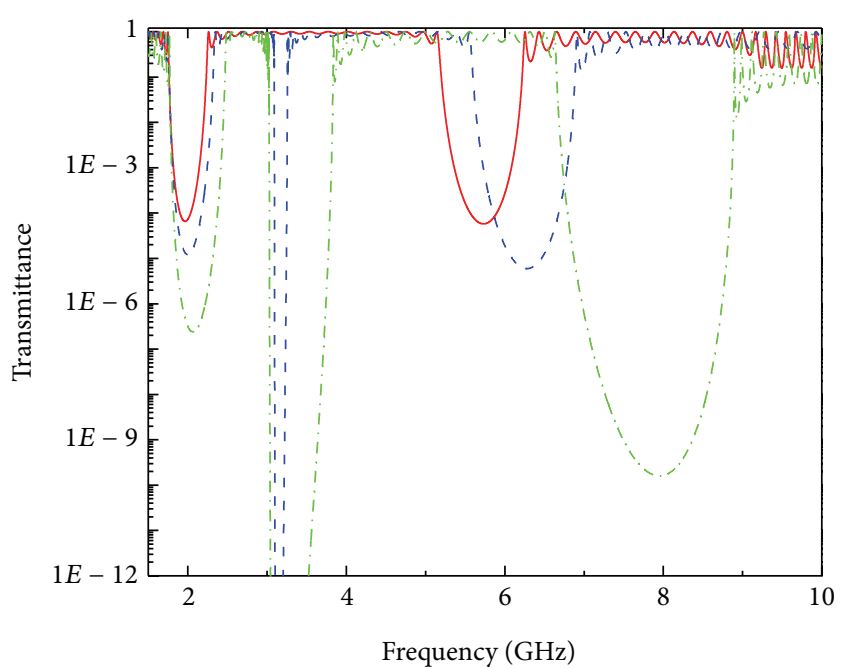

$$
\begin{aligned}
& \text { TE mode } \\
& -\theta_{0}=0^{\circ} \\
& --\theta_{0}=30^{\circ} \\
& --\theta_{0}=60^{\circ}
\end{aligned}
$$

(a)

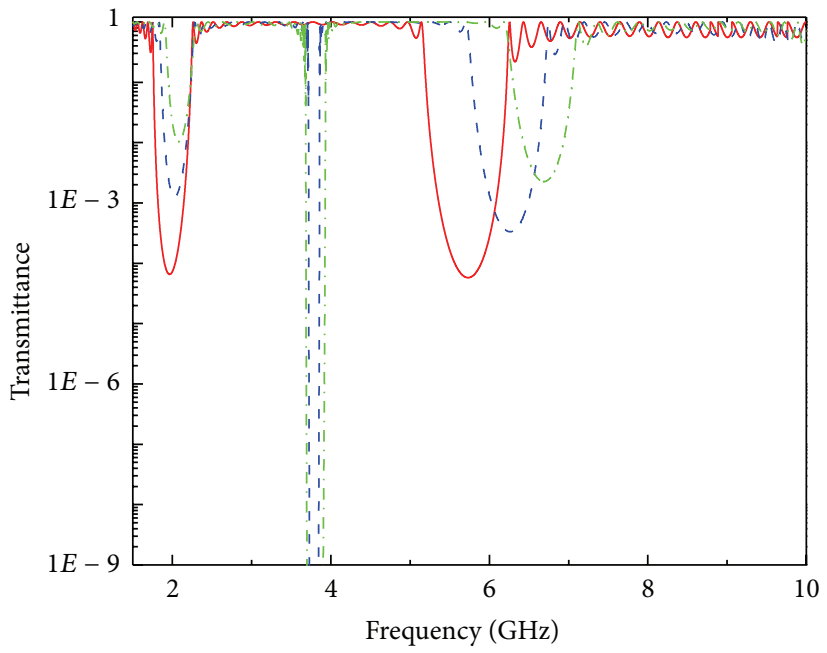

TM mode

$$
\begin{aligned}
-\theta_{0} & =0^{\circ} \\
---\theta_{0} & =30^{\circ} \\
--\theta_{0} & =40^{\circ}
\end{aligned}
$$

(b)

FIGURE 3: Transmission spectra for the 1D ternary MPC structure for different angles of incidence with $\gamma=0.2 \times 10^{-3} \mathrm{GHz}$ (a) TE and (b) TM waves.

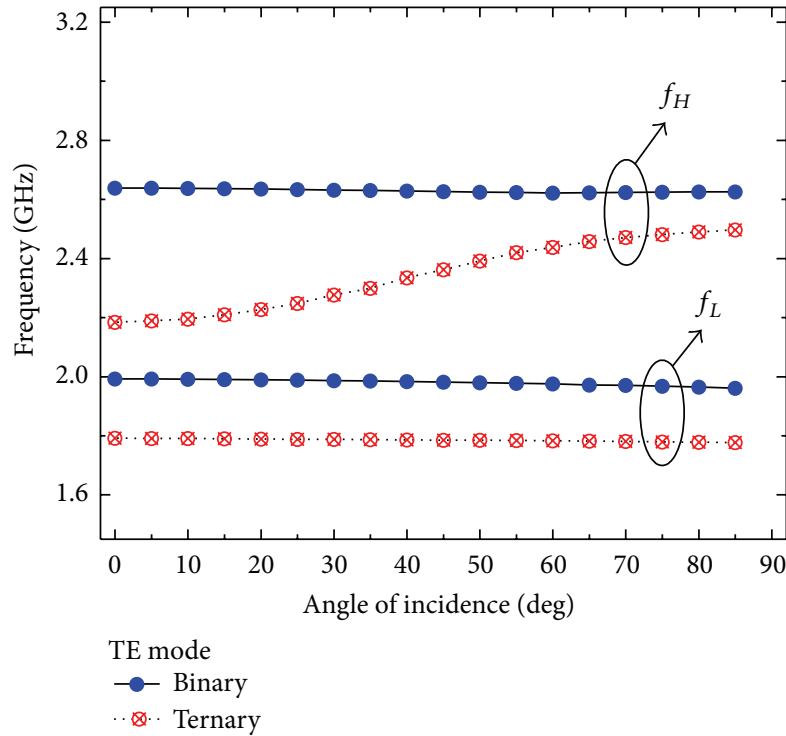

(a)

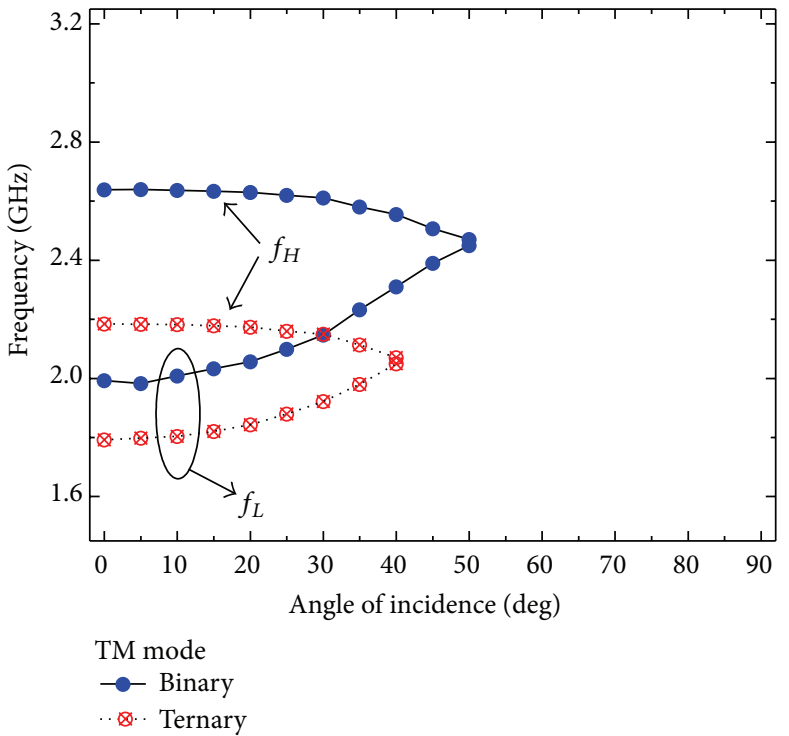

(b)

FIGURE 4: The lower and higher frequencies of the zero- $\bar{n}$ gap as a function of angle of incidence for both binary and ternary structures: (a) TE and (b) TM waves.

is sensitive to the angle of incidence and increases as the incidence angle increases, while the left edge of the band gap is nearly invariant. Moreover, the width of the zero- $\bar{n}$ gap of both binary and ternary structures is very sensitive to the incidence angle for TM wave, and the gap disappears for incidence angles greater than $50^{\circ}$ in the binary structure as reported in $[10,12]$. The gap disappears for incidence angles greater than $40^{\circ}$ for the ternary structure.
The lower and the higher frequencies of the zero- $\mu$ and zero- $\varepsilon$ gaps as a function of incidence angle, for both binary and ternary structures, are shown in Figures 5(a) and 5(b), respectively. The width of the zero- $\mu$ and zero- $\varepsilon$ gaps increases faster for the binary structure than the ternary ones. As it is clearly seen, the gaps appear at the same frequency for both binary and ternary structures; in addition, the figures indicate the upper edge of the gaps, so the width of the gaps is very 


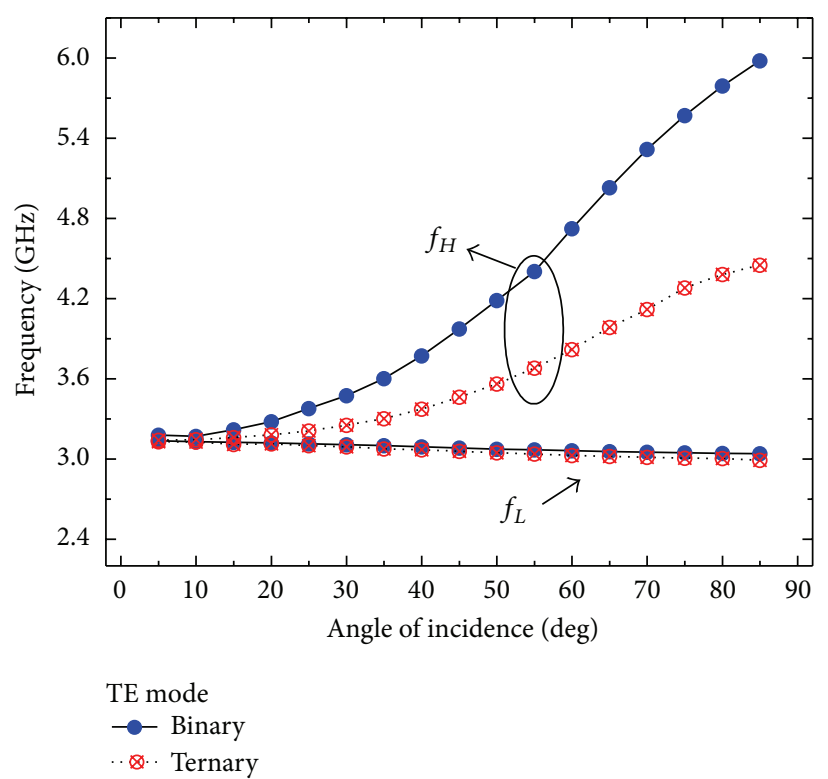

(a)

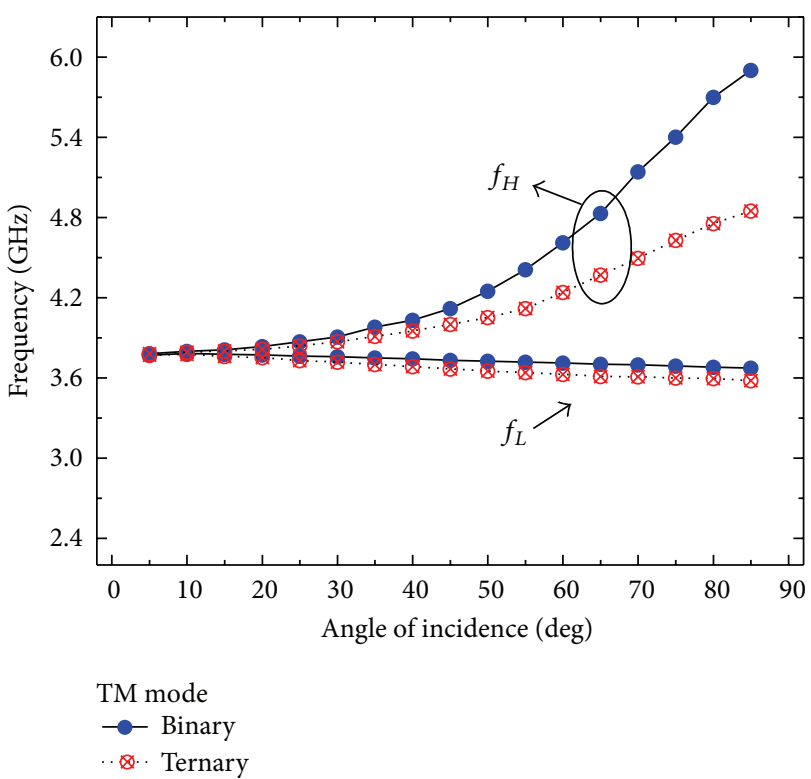

(b)

Figure 5: The lower and higher frequencies of the (a) zero- $\mu$ and (b) zero- $\varepsilon$ gaps as a function of angle of incidence for both binary and ternary structures.

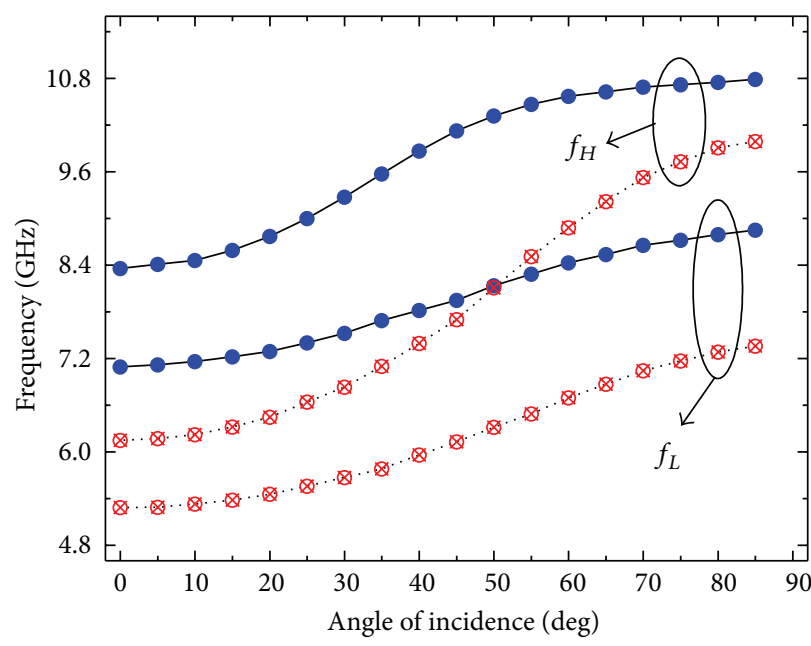

$$
\begin{aligned}
& \text { TE mode } \\
& - \text { Binary } \\
& - \text { Ternary }
\end{aligned}
$$

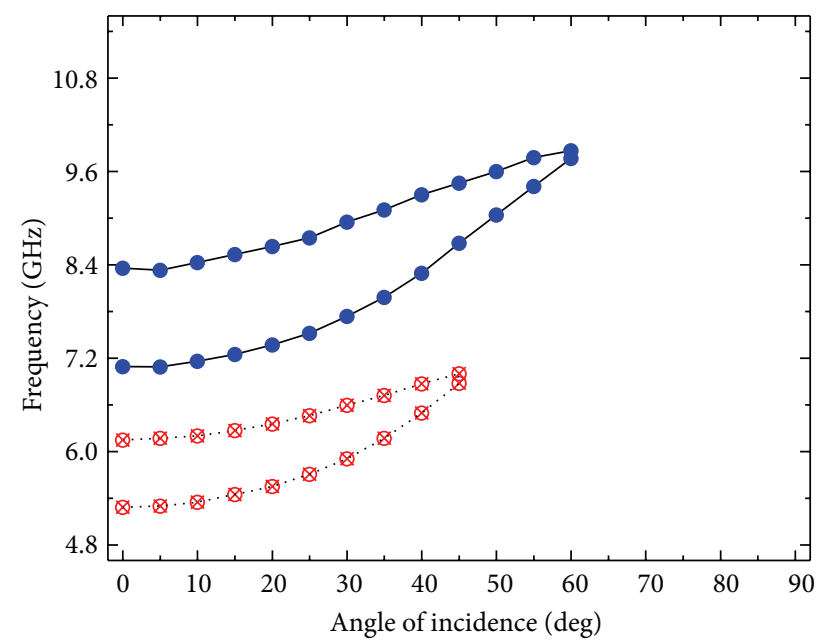

TE mode

$$
\bullet \text { Binary }
$$

\$. Ternary

(a)

(b)

FIGURE 6: The lower and higher frequencies of the Bragg gap as a function of angle of incidence for both binary and ternary structures: (a) TE and (b) TM waves.

sensitive to the incidence angle, while the lower edge of the gaps is nearly insensitive.

The $f_{L}$ and $f_{H}$ frequencies of the Bragg gap of the binary and ternary structures, as a function of incidence angle for TE and TM waves, are shown in Figures 6(a) and 6(b), respectively. It is seen that the Bragg gap like the zero- $\bar{n}$ gap appears in the lower frequencies for the ternary structure. Moreover, $f_{L}$ and $f_{H}$ shift to the higher frequencies as the incidence angle increases, and as it is seen from Figure 6(b), the gap for TM polarized wave disappears for incidence angles greater than $60^{\circ}$ for the binary and $45^{\circ}$ for the ternary structures.

\section{Conclusion}

The numerical results show that including the loss factor in the permittivity and permeability of the DNG layer the changes in transmission spectrum of TE wave in $1 \mathrm{D}$ ternary 
and binary MPCs behave similar to different incidence angles. The zero- $\bar{n}$, zero- $\mu$, and the Bragg gaps become wider and deeper as the angle of incidence increases. In addition, by enhancing the angle of incidence, the lower frequencies of the zero- $\bar{n}$ and zero- $\mu$ gaps are nearly invariant in both ternary and binary MPCs, but the Bragg gap increases. Moreover, the upper edge of all three band gaps increases. Such behaviors were not clearly observed in the zero- $\bar{n}$ gap. The transmission spectrum for TM polarized wave is rather different. It is found that, in both structures, as the angle of incidence increases, the width and the depth of the zero- $\varepsilon$ gap increase, while for the zero- $\bar{n}$ gap and for the angles of incidence greater than $40^{\circ}$ for the ternary and $50^{\circ}$ for the binary structures, the zero$\bar{n}$ gap disappears. Similar behavior is observed for the Bragg gap for the angles of incidence greater than $45^{\circ}$ and $60^{\circ}$ for binary and ternary structures, respectively. For TM waves, the lower frequency of the zero- $\bar{n}$ and Bragg gaps increases, but the zero- $\varepsilon$ gap decreases as the angle of incidence increases in both binary and ternary structures. The higher edge of the zero- $\varepsilon$ and Bragg gaps increases, while it decreases for the zero- $\bar{n}$ gap. It is interesting to note that the difference between the lower and higher frequencies of all the gaps for TM wave in the binary MPC is larger than that in the ternary MPC as all the figures show. To recapitulate what was said before, (i) the width of the band gaps decreases as a dielectric layer is added to the binary structure, (ii) the zero- $\bar{n}$ and Bragg gaps appear at the lower frequencies in the ternary structure, and (iii) the zero- $\varepsilon$ and zero- $\mu$ gaps appear at the same frequencies in both structures. Finally, the results of the present study could be employed in designing new edge filters, waveguides, and other optical devises in microwave engineering. In addition, detailed analysis of the band gaps in ternary metamaterial photonic crystal will certainly provide useful information for designing new types of transmission filter. This filter has a superior feature of ternary structure, which is not seen in the usual binary photonic crystals.

\section{Conflict of Interests}

The authors declare that there is no conflict of interests regarding the publication of the paper.

\section{Acknowledgment}

Alireza Aghajamali would like to acknowledge his gratitude to Parisa Shams for her help and useful discussion.

\section{References}

[1] E. Yablonovitch, "Inhibited spontaneous emission in solid-state physics and electronics," Physical Review Letters, vol. 58, no. 20, pp. 2059-2062, 1987.

[2] S. John, "Strong localization of photons in certain disordered dielectric superlattices," Physical Review Letters, vol. 58, no. 23, pp. 2486-2489, 1987.

[3] S. Noda, A. Chutinan, and M. Imada, "Trapping and emission of photons by a single defect in a photonic bandgap structure," Nature, vol. 407, no. 6804, pp. 608-610, 2000.
[4] S. Massaoudi, A. De Lustrac, and I. Huynen, "Properties of metallic photonic band gap material with defect at microwave frequencies: calculation and experimental verification," Journal of Electromagnetic Waves and Applications, vol. 20, no. 14, pp. 1967-1980, 2006.

[5] B. Mandal and A. R. Chowdhury, "Spatial soliton scattering in a quasi phase matched quadratic media in presence of cubic nonlinearity," Journal of Electromagnetic Waves and Applications, vol. 21, no. 1, pp. 123-135, 2007.

[6] Q.-R. Zheng, B.-Q. Lin, Y.-Q. Fu, and N.-C. Yuan, "Characteristics and applications of a novel compact spiral electromagnetic band-gap (EBG) structure," Journal of Electromagnetic Waves and Applications, vol. 21, no. 2, pp. 199-213, 2007.

[7] E. Yablonovitch, T. J. Gmitter, and K. M. Leung, "Photonic band structure: the face-centered-cubic case employing nonspherical atoms," Physical Review Letters, vol. 67, no. 17, pp. 2295-2298, 1991.

[8] J. D. Joannopoulos, S. G. Johnson, J. N. Winn, and R. D. Meada, Photonic Crystals: Modeling the Flow of Light, Princeton University Press, Princeton, NJ, USA, 2008.

[9] J. Li, L. Zhou, C. T. Chan, and P. Sheng, "Photonic band gap from a stack of positive and negative index materials," Physical Review Letters, vol. 90, no. 8, Article ID 083901, 2003.

[10] A. Aghajamali and M. Barati, "Effects of normal and oblique incidence on zero- $\bar{n}$ gap in periodic lossy multilayer containing double-negative materials," Physica B, vol. 407, no. 8, pp. 12871291, 2012.

[11] H. Daninthe, S. Foteinopoulou, and C. M. Soukoulis, "Omnireflectance and enhanced resonant tunneling from multilayers containing left-handed materials," Photonics and Nanostructures, vol. 4, no. 3, pp. 123-131, 2006.

[12] S. K. Awasthi, A. Mishra, U. Malaviya, and S. P. Ojha, "Wave propagation in a one-dimensional photonic crystal with metamaterial," Solid State Communications, vol. 149, no. 33-34, pp. 1379-1383, 2009.

[13] Y. Xiang, X. Dai, and S. Wen, "Omnidirectional gaps of onedimensional photonic crystals containing indefinite metamaterials," Journal of the Optical Society of America B, vol. 24, no. 9, pp. 2033-2039, 2007.

[14] H. Jiang, H. Chen, H. Li, Y. Zhang, and S. Zhu, "Omnidirectional gap and defect mode of one-dimensional photonic crystals containing negative-index materials," Applied Physics Letters, vol. 83, no. 26, pp. 5386-5388, 2003.

[15] E. Silvestre, R. A. Depine, M. L. Martínez-Rieci, and J. A. Monsoriu, "Role of dispersion on zero-average-index bandgaps," Journal of the Optical Society of America B, vol. 26, no. 4, pp. 581-586, 2009.

[16] J. A. Monsoriu, R. A. Depine, M. L. Martínez-Ricci, and E. Silvestre, "Interaction between non-Bragg band gaps in ID metamaterial photonic crystals," Optics Express, vol. 14, no. 26, pp. 12958-12967, 2006.

[17] R. A. Depine, M. L. Martínez-Ricci, J. A. Monsoriu, E. Silvestre, and P. Andrés, "Zero permeability and zero permittivity band gaps in 1D metamaterial photonic crystals," Physics Letters A, vol. 364, no. 3-4, pp. 352-355, 2007.

[18] S. K. Singh, J. P. Pandey, K. B. Thapa, and S. P. Ojha, "Some new band gaps and defect modes of 1D photonic crystals composed of metamaterials," Solid State Communications, vol. 143, no. 4-5, pp. 217-222, 2007.

[19] A. Aghajamali and M. Barati, "Properties of defect modes in periodic lossy multilayer with negative-index-materials," Communications in Theoretical Physics, vol. 60, pp. 80-86, 2013. 
[20] A. Aghajamali, M. Hayati, C. J. Wu, and M. Barati, "Properties of the defect modes in 1D lossy photonic crystals containing two types of negative-index-materials defects," Journal of Electromagnetic Waves and Applications, vol. 27, pp. 2317-2329, 2013.

[21] A. Aghajamali, B. Javanmardi, M. Barati, and C. J. Wu, "Defect modes properties in periodic lossy multilayer containing negative index materials with symmetric and asymmetric geometries structures," Optik, vol. 125, pp. 839-843, 2014.

[22] M. Born and E. Wolf, Principles of Optics: Electromagnetic Theory of Propagation, Interference and Diffraction of Light, Cambridge University Press, Cambridge, UK, 2005. 

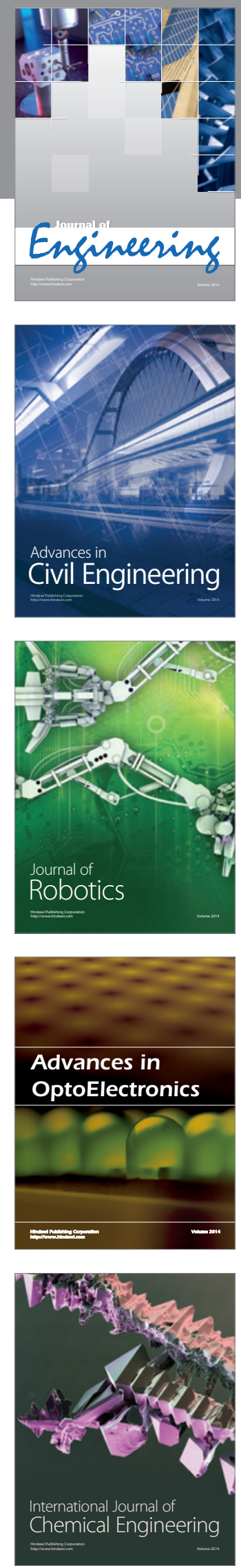

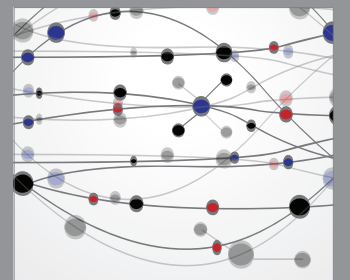

The Scientific World Journal
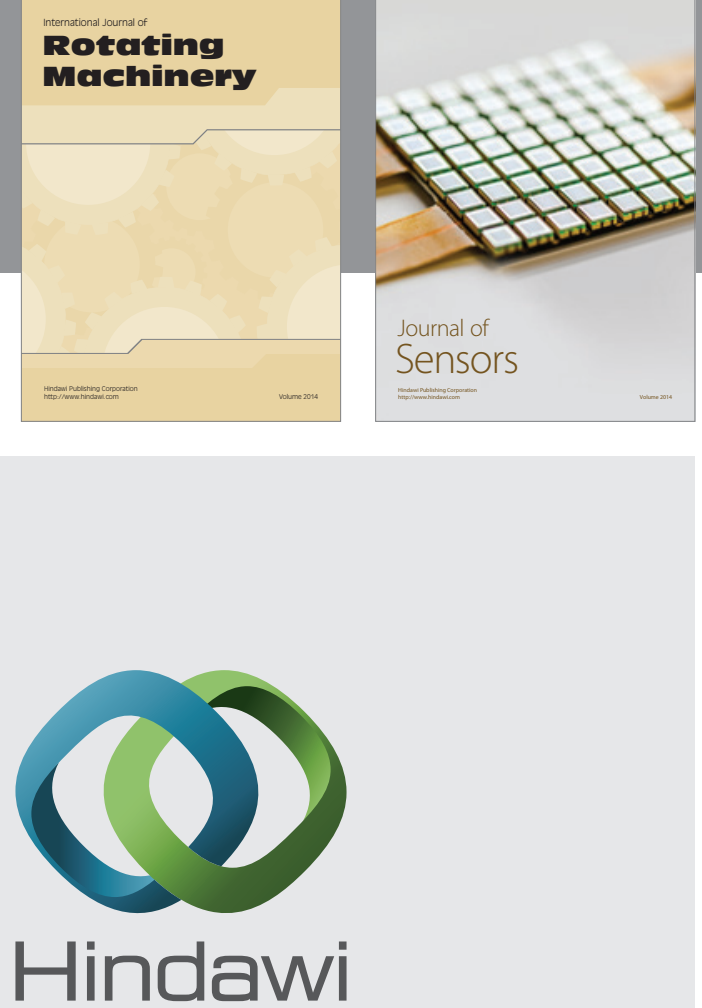

Submit your manuscripts at http://www.hindawi.com
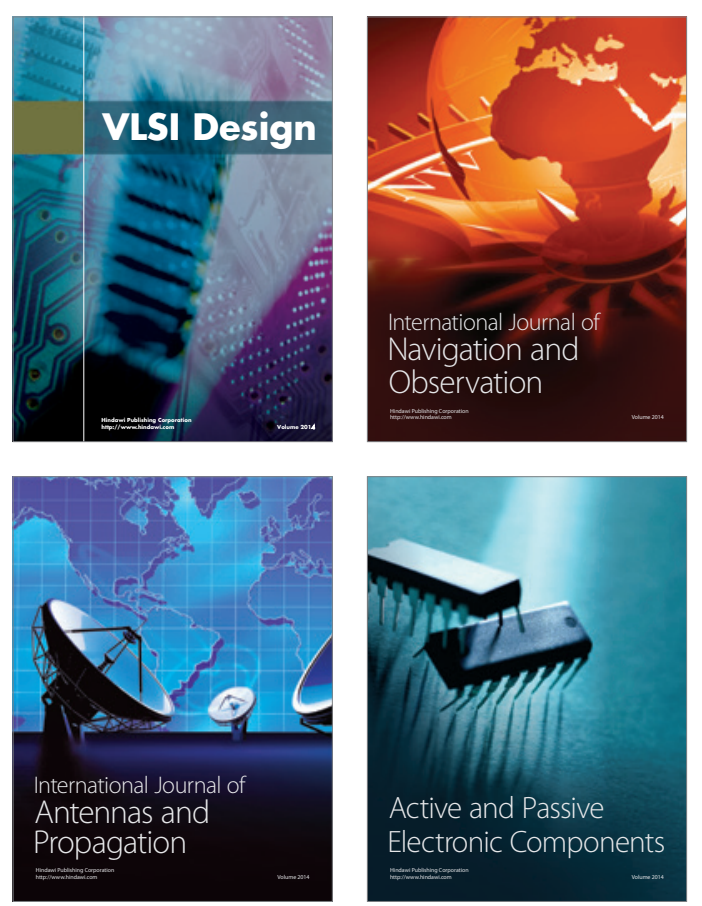
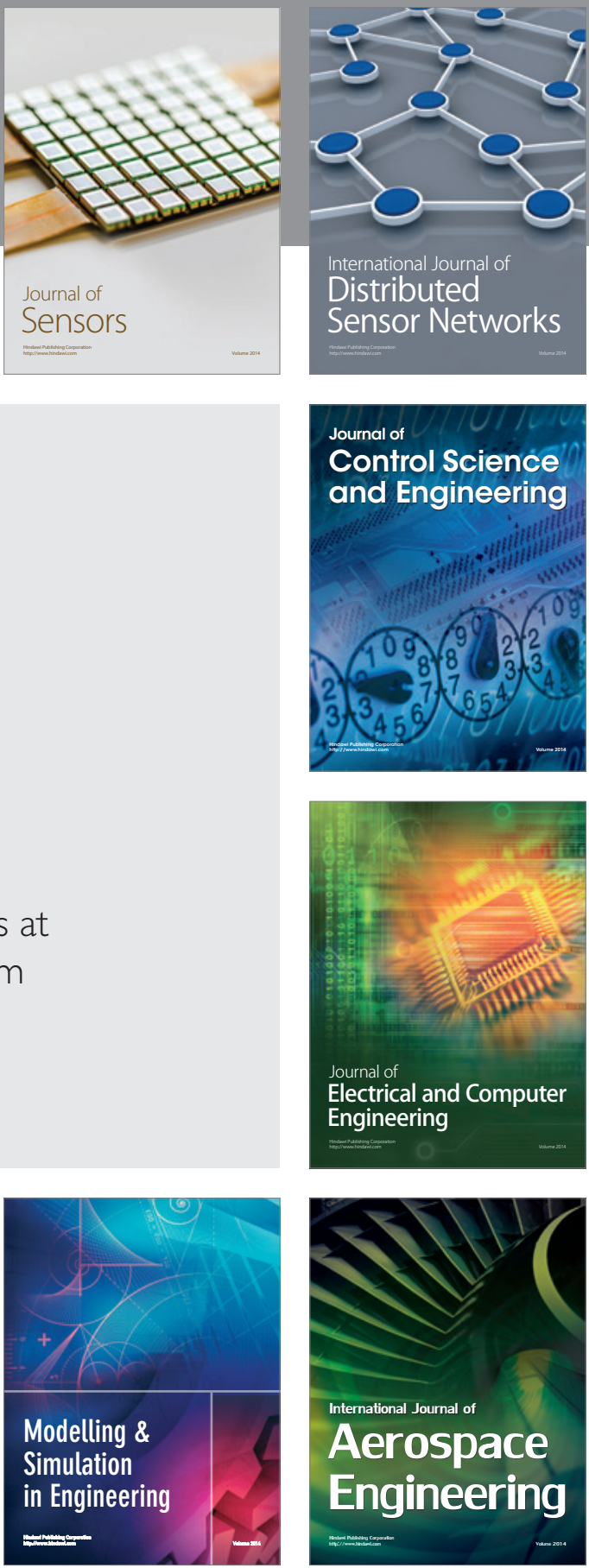

Journal of

Control Science

and Engineering
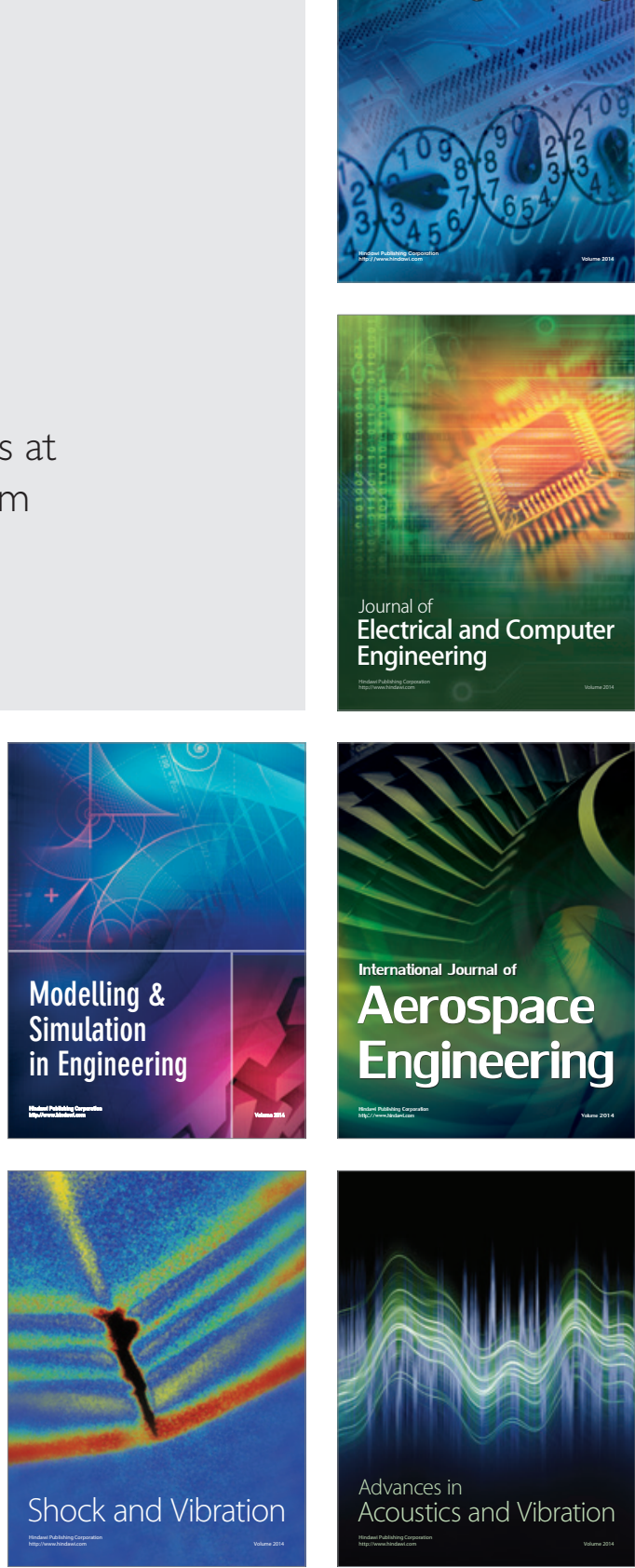